Mesopotamia J. of Agric.

Vol. (41) No. (1) 2013
ISSN: 2224-9796 (Online) ISSN: $1815-316$ X (Print)
مجـلـة زر اعــة الـــر افديـنـ

المجلد (41) العدد (1) 2013

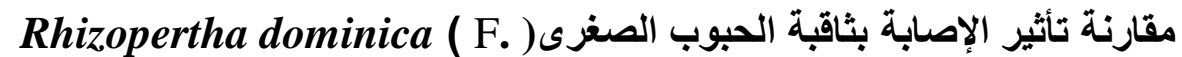 لعشرة أصناف من الحنطة قبل وبعد عملية الدراس (Bostrichidae:Coleoptera )
}

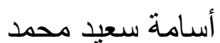

قسم وقاية النبات / كلية الزر اعة و الغابات / جامعة الموصل

Osa1953@Yahoo.com

\section{الخلاصة}

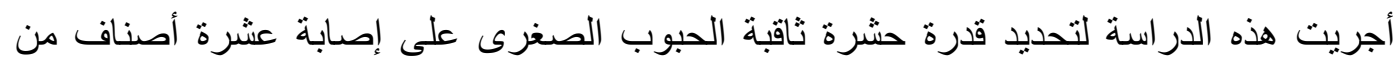

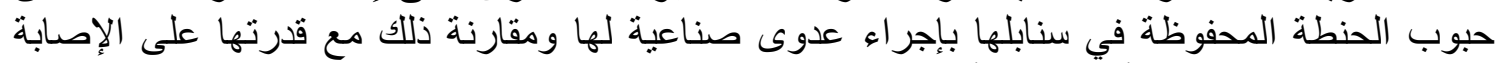

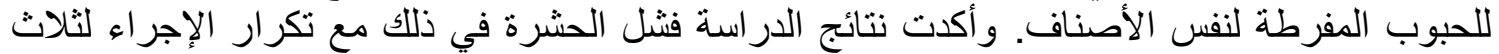

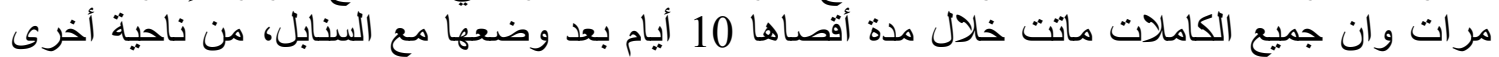
كانت نسبة الإصابة للحبوب المفرطة لنفس الأصناف منباينة بلغت في معدلها 52.3 \% 52.3 \%

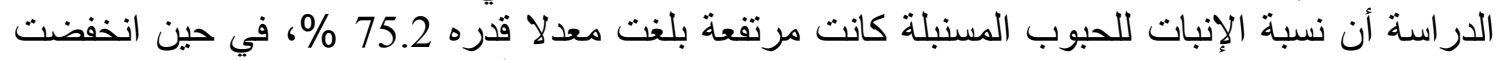
نسبة إنبات الحبوب المفرطة معنويا وبلغت 32.3 \% في المعدل. كما أدت الإصابة بالحشرة إلى في انخفاض

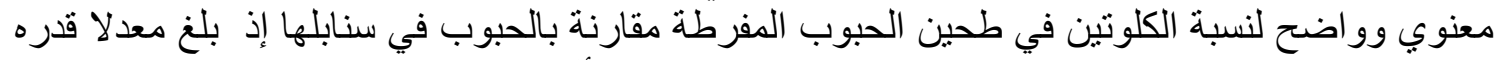
19.9 \% وهي نسب منخفضة وغير مقبولة لصناعة الخبز ألمختبري (اللوف) في حين كانت نسبة فئة

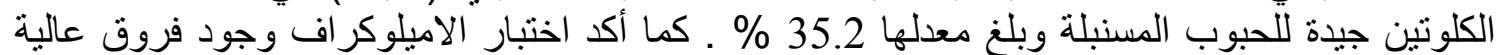
المعنوية بين قيم اللزوجة القصوى للحبوب في سنابلها مقارنة بالمصابة إذ كانت عالية جدا في الأخيرة

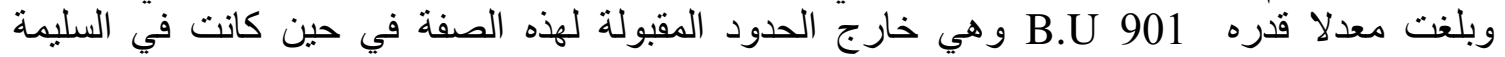

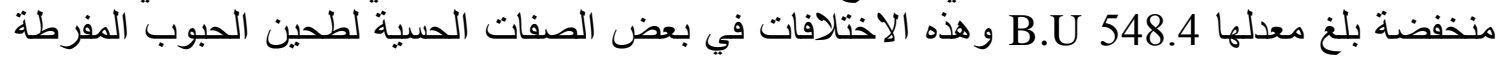

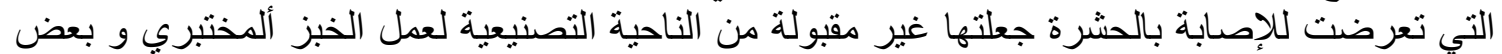

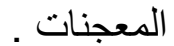

كلمات دالة: ثاقبة الحبوب الصغرى، أصناف الحنطة ، الصفات الريولوجية.

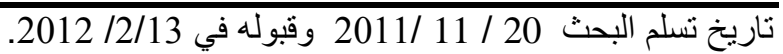

\section{المقدمة}

يمثل القمح واحدا من أكثر السلع الغذائية أهمية وانتشارا على النطاق المحلى والعالمي بالنسبة

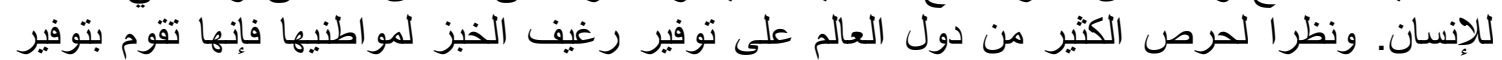

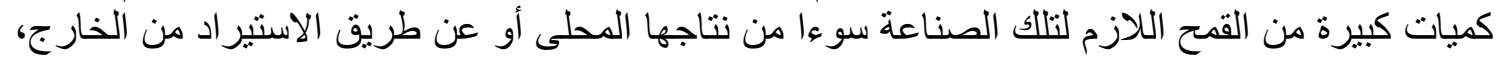

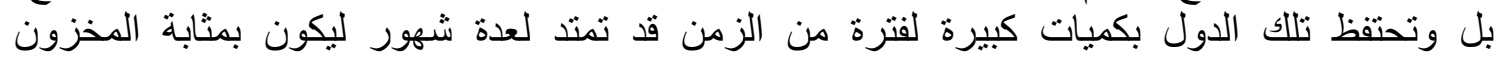

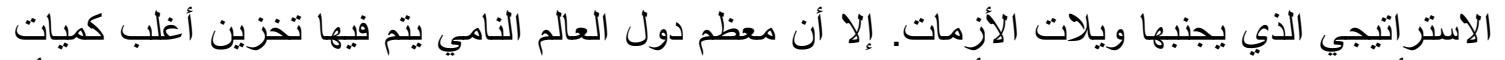

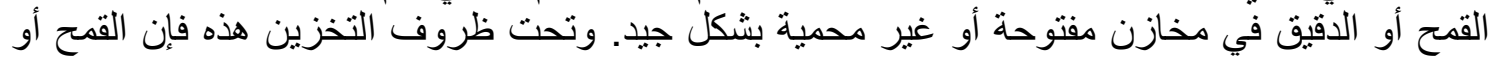

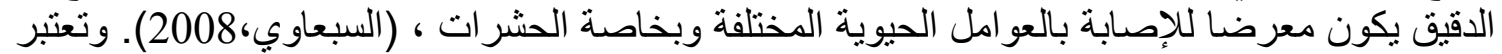
حشرة ثاقبة الحبوب الصغرى وأسمها العلمي Rhizopertha dominica والتي تنبع العائلة Bostrichidae

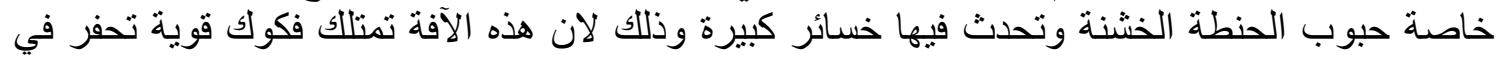
الحبوب الصلبة وتتغذى عليها وتدخل يرقاتها داخل الحبة وتتغذى على محتوياتها و لا تبقي منها سوى الته القثور كما أنها تستهلك أكثر مما تحتاج إليه في غذائها علاوة على قدرتها على ثلى ثلب الحبوب الأكثر

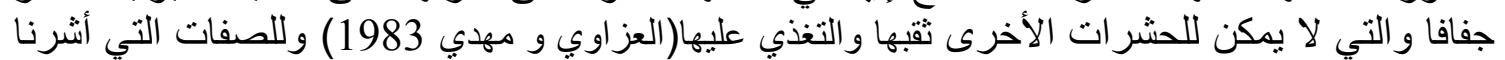
إليها فقد هدف البحث إلى اختبار قدرة هذه الآفة على إصابة الحبوب المابكة المحفوظة في سنابلها

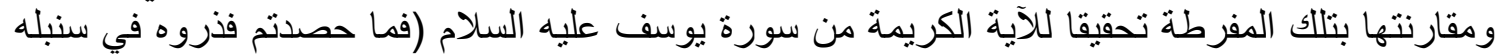

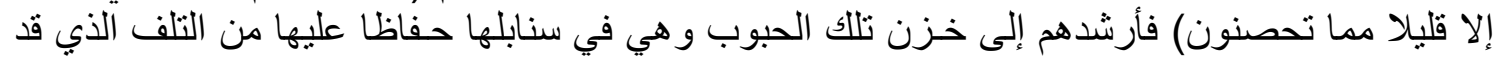

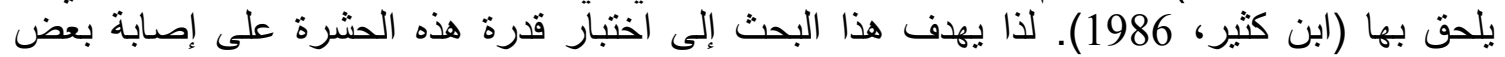

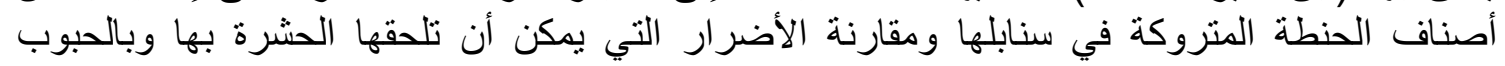


Mesopotamia J. of Agric.

Vol. (41) No. (1) 2013
ISSN: 2224-9796 (Online) ISSN: $1815-316$ X (Print)

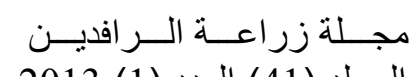

المجلا (41) العدد (1) 2013

المفرطة ، للخروج بتوصيات تخدم عملية الخزن في حالة وجود هكذا آفات يخشى منها ومما قد تسببه من

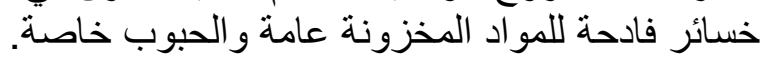

\section{مواد البحث وطر ائقه}

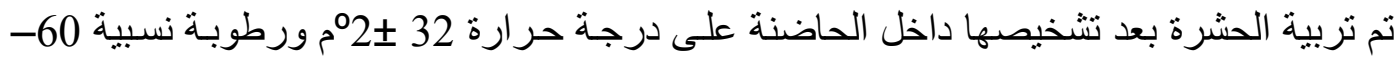

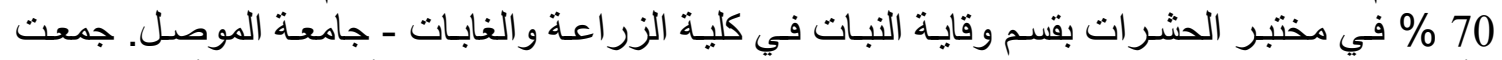

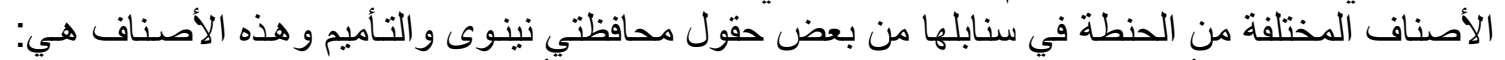

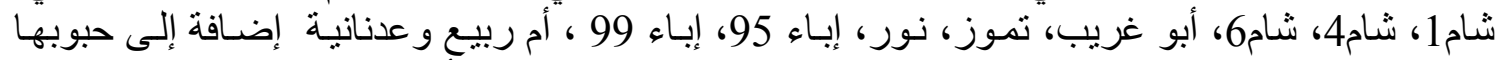

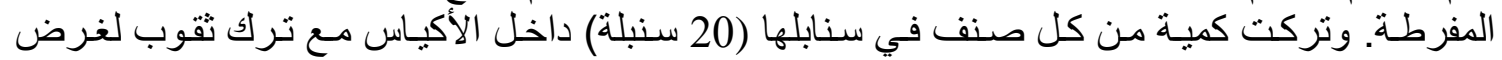

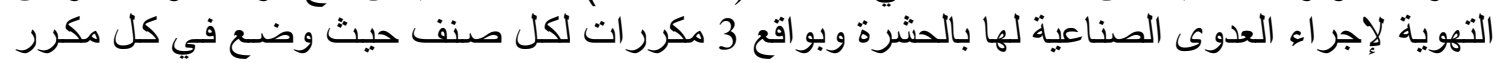

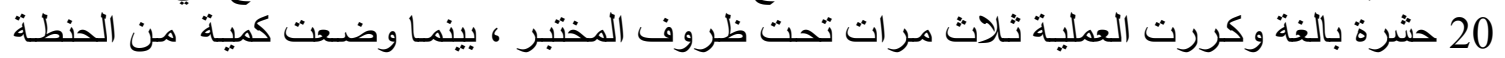

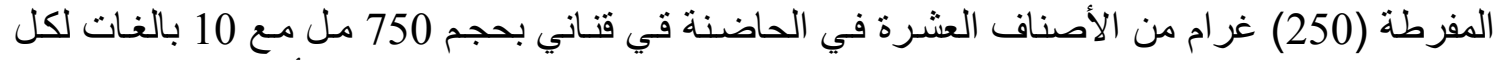

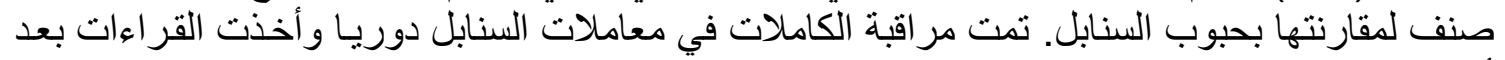

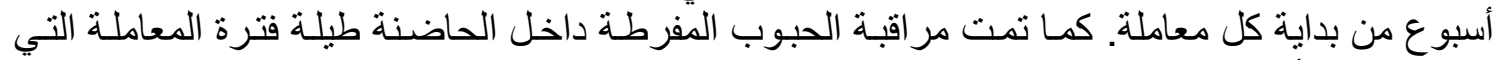

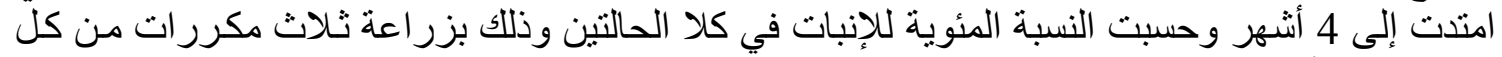

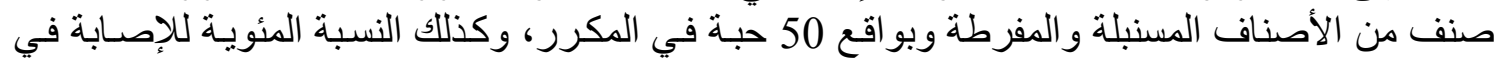

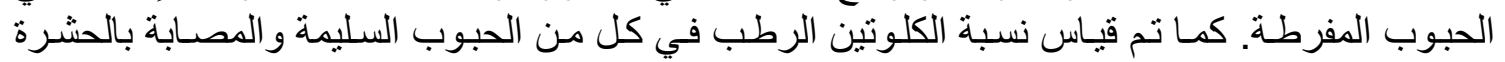

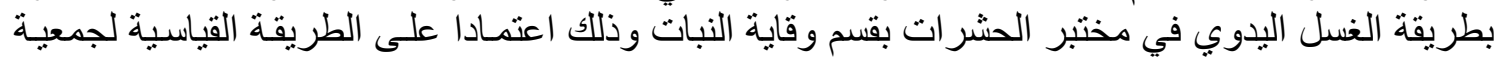

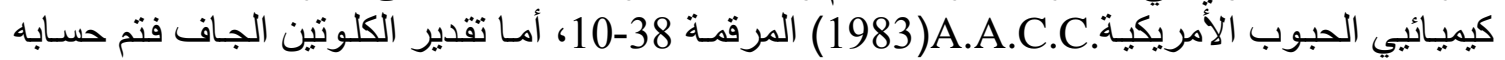

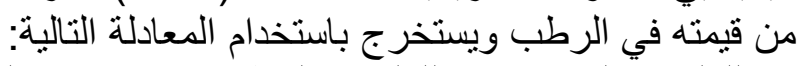

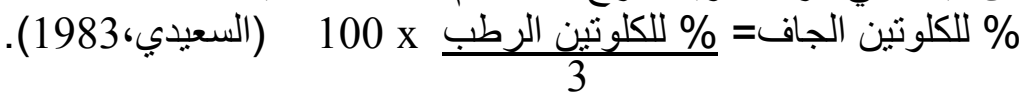

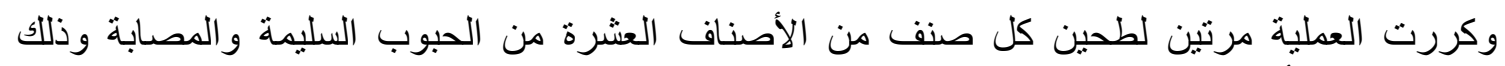

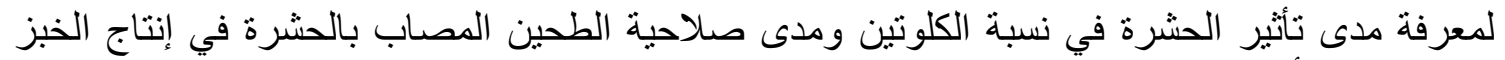

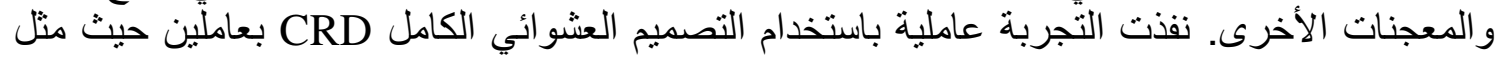

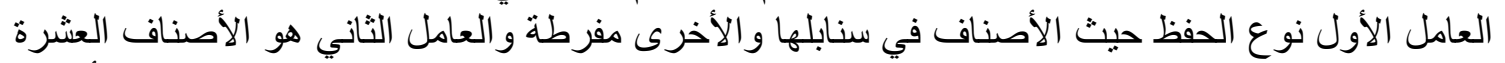

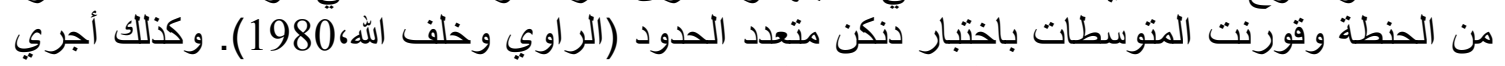

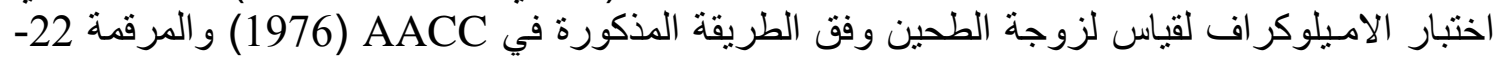

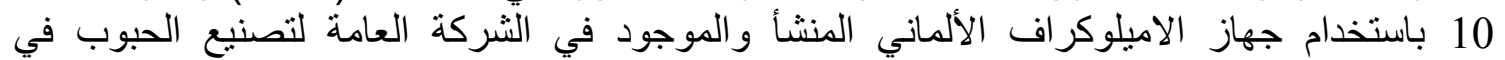

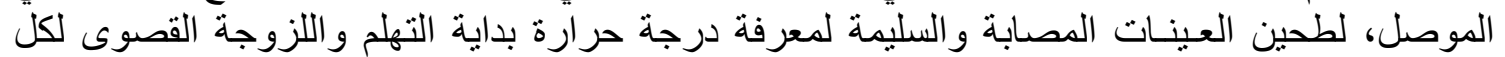

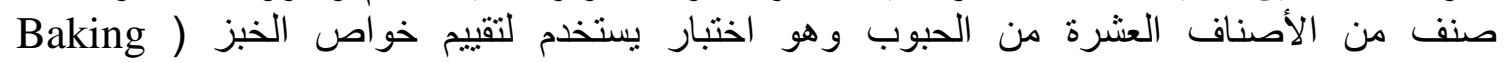
و و يعتمد بدرجة كبيرة على خواص التهلم Groperties

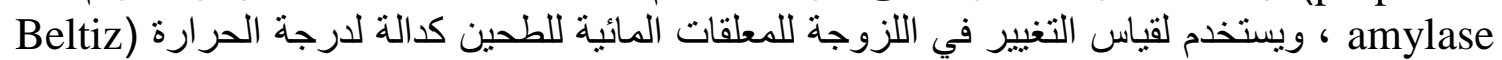

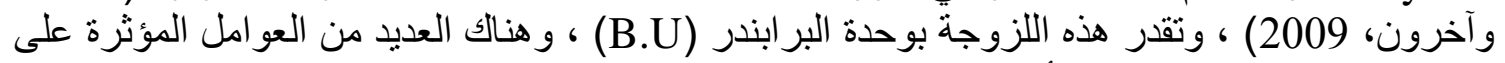

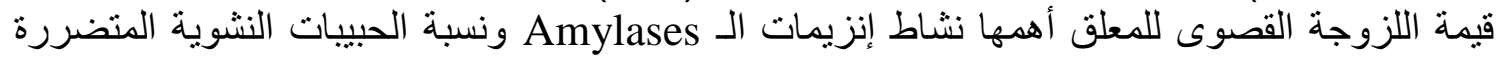
(1990 Stear ؛ 1988 Pomeranz)

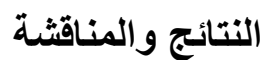

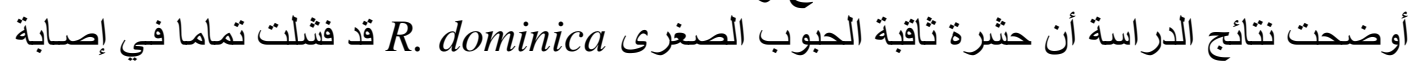

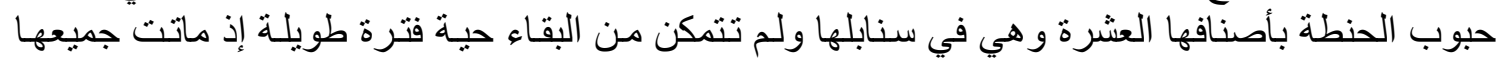

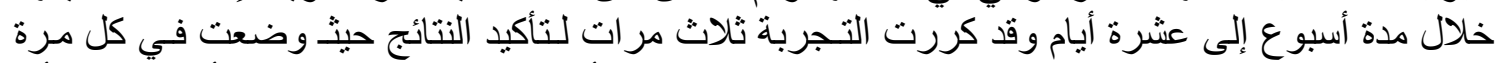

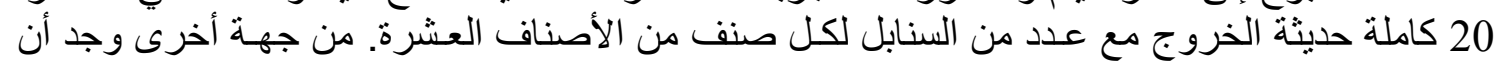

الحشرة وعند وضعها مع الحبوب المفرطة لنفس الأصناف داخل الحاضنة ولفترة 4 أثنهر قد فتكت بها

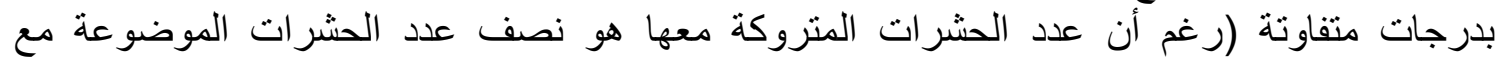


Mesopotamia J. of Agric.

Vol. (41) No. (1) 2013
ISSN: 2224-9796 (Online)

ISSN: $1815-316 \mathrm{X}$ (Print)

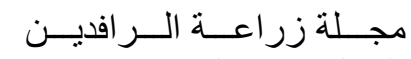

المجلا (41) العدد (1) 2013

السنابل) مما اثر وبشكل و اضح و عالي المعنوية على نسب إنباتها و التي تراوحت بين 1 \% \% للصنف أبو

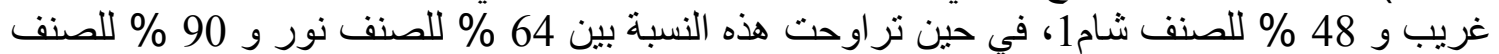

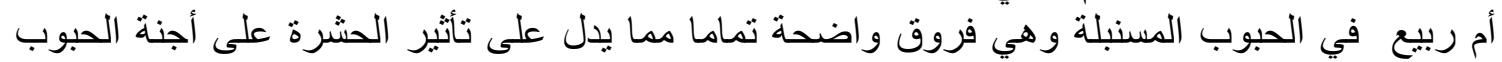

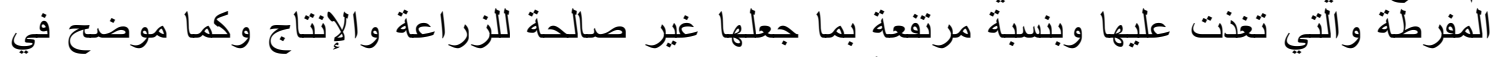
الجدول (1) إذ اختلفت النسب في جميع الأصناف السليمة عن المصابة اختلافا عالي المعنوية.

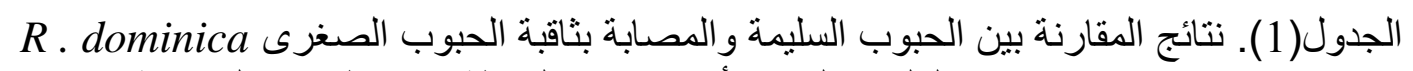
في نسب الإنبات و الكلوتين لعشرة أصناف من الحنطة تحت ظروف الحت الحاضنة.

Table(1). The comparison between sound and infested seed grains by Lesser grain borer $R$. dominica in the percentages of germination and gluten for 10 varieties of wheat under incubator conditions.

\begin{tabular}{|c|c|c|c|c|c|c|c|}
\hline \multirow{2}{*}{$\begin{array}{c}\text { Infestation } \\
\text { للإصابة \% }\end{array}$} & \multicolumn{2}{|c|}{$\begin{array}{l}\text { \% الكلوتين الجاف\% } \\
\text { Dry gluten\% }\end{array}$} & \multicolumn{2}{|c|}{$\begin{array}{l}\text { \% للكلوتين الرطب \% \% } \\
\text { wet gluten \% }\end{array}$} & \multicolumn{2}{|c|}{$\begin{array}{l}\text { \% معل \% للإنبات } \\
\text { Germination }\end{array}$} & \multirow[t]{2}{*}{$\begin{array}{r}\text { الأصناف } \\
\text { Varieties }\end{array}$} \\
\hline & $\begin{array}{l}\text { Infested } \\
\text { Inved }\end{array}$ & $\begin{array}{l}\text { السليمة } \\
\text { Sound }\end{array}$ & $\begin{array}{l}\text { المصابة } \\
\text { Infested }\end{array}$ & $\begin{aligned} \text { السليمة } \\
\text { Sound }\end{aligned}$ & $\begin{array}{c}\text { المصابة } \\
\text { Infested }\end{array}$ & $\begin{array}{r}\text { السليمة } \\
\text { Sound }\end{array}$ & \\
\hline 24 & 8.3 & 13.3 & $25 \mathrm{gh}$ & $40 \mathrm{ab}$ & $48 \mathrm{~d}$ & $76 \mathrm{bc}$ & Sham 1 شام1 \\
\hline 40 & 6.0 & 11.3 & $21 \mathrm{i}$ & $34 \mathrm{~d}$ & $42 \mathrm{de}$ & $80 a b c$ & Sham 4 شام4 \\
\hline 52 & 8.7 & 10.3 & $24 \mathrm{~h}$ & $31 \mathrm{c}$ & $36 \mathrm{def}$ & $72 \mathrm{c}$ & شام 6 Sham 6 \\
\hline 99 & -- & 13 & - & $39 \mathrm{~b}$ & $1 \quad \mathrm{i}$ & $74 \mathrm{c}$ & Abu-Graib \\
\hline 38 & 6.0 & 10.7 & $18 \mathrm{j}$ & $32 \mathrm{e}$ & $40 \mathrm{de}$ & $68 \mathrm{c}$ & تموز2 2 \\
\hline 32 & 8.0 & 10.7 & $24 \mathrm{~h}$ & $32 \mathrm{e}$ & $34 \mathrm{fg}$ & $64 \mathrm{c}$ & Noor \\
\hline 42 & 9.0 & 10.3 & $27 \mathrm{f}$ & $31 \mathrm{e}$ & $32 \mathrm{fg}$ & $88 \mathrm{ab}$ & Iba’a $95{ }^{95}$ \\
\hline 52 & 8.7 & 12.0 & $26 \mathrm{fe}$ & $36 \mathrm{c}$ & $34 \mathrm{fg}$ & $72 \mathrm{c}$ & Iba'a 99 إباء 99 \\
\hline 74 & 5.3 & 13.7 & $16 \mathrm{k}$ & $41 \mathrm{a}$ & $32 \mathrm{fg}$ & $90 \mathrm{a}$ & Om-Rabee \\
\hline 70 & 6.0 & 12.0 & $18 \mathrm{j}$ & $36 \mathrm{c}$ & $24 \mathrm{~h}$ & $68 \mathrm{c}$ & عدنانية Adnania \\
\hline 52.3 & -- & -- & $19.9 \mathrm{~b}$ & $35.2 \mathrm{a}$ & $32.3 \mathrm{~b}$ & $75.2 \mathrm{a}$ & Mean \\
\hline
\end{tabular}

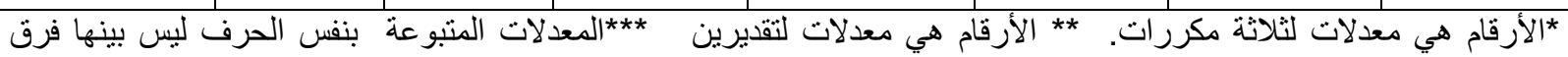

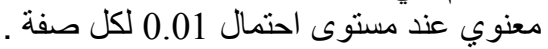
*The numbers are averages of 3 rep. ** The numbers are averages of 2 rep. ***The averages taking the same letters have no sig. differences between others.

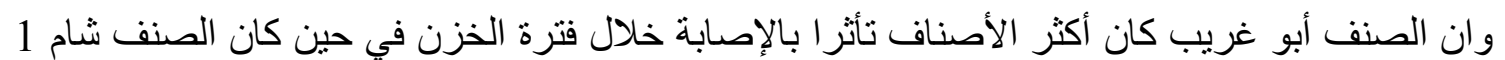

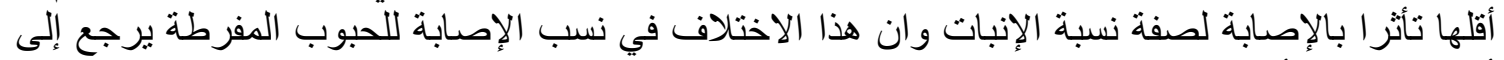

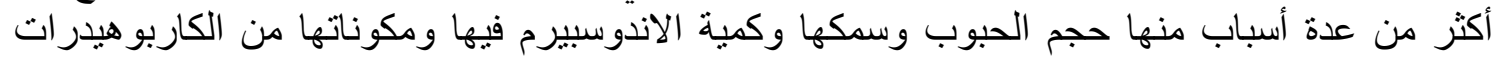

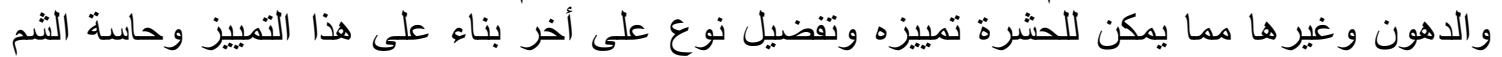

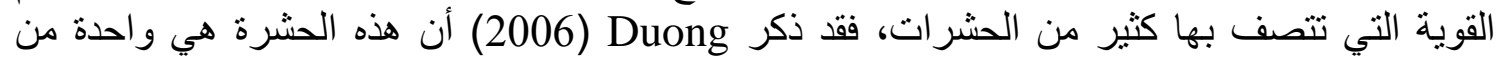

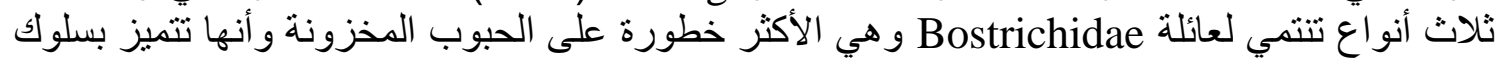

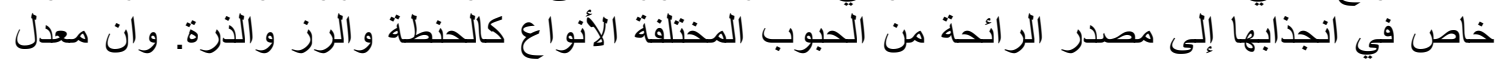

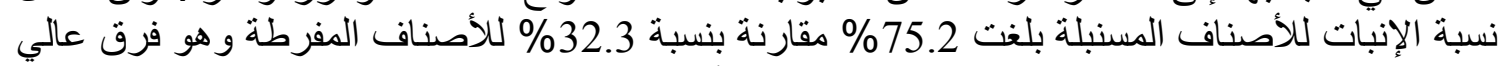
المعنوية، مما يدل على عدم تحمل الحبوب المفرطة بأصنافها المختلفة للإصابة بهذه الآفة عند تخزينها 
Mesopotamia J. of Agric.

Vol. (41) No. (1) 2013
ISSN: 2224-9796 (Online) ISSN: 1815-316 X (Print)

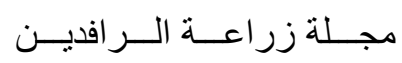

2013 المجلد (41) العدد (1)

و تعرضها لها أثناء فترة الخزن القصيرة. و عند تقدير نسبة الكلوتين في طحين كلا النوعين من الحبوب

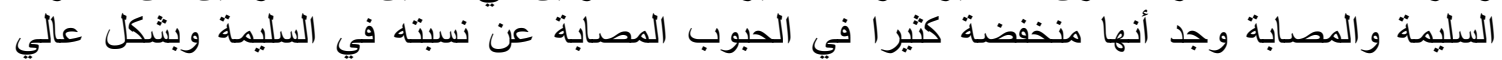

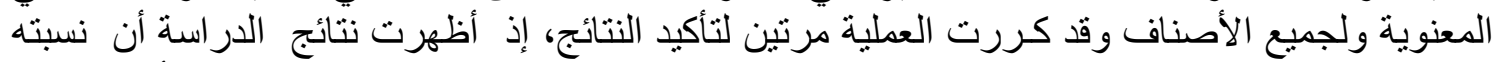

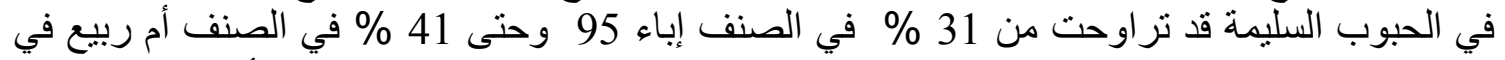

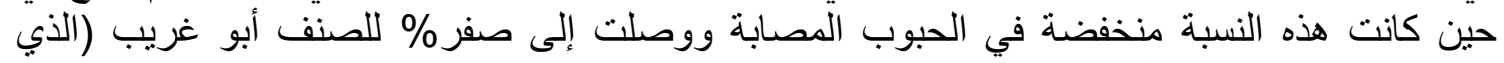

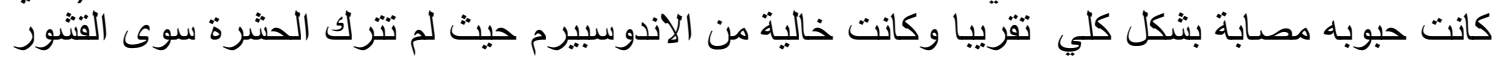

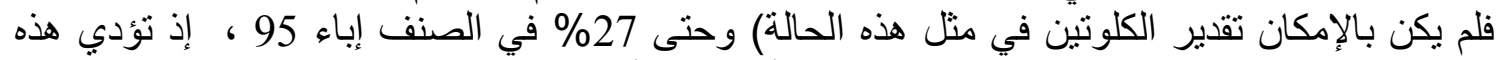

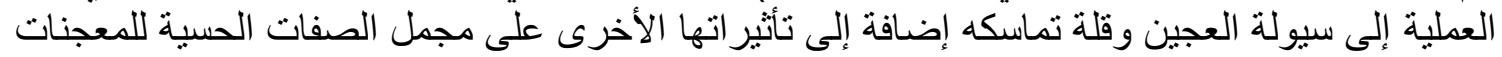

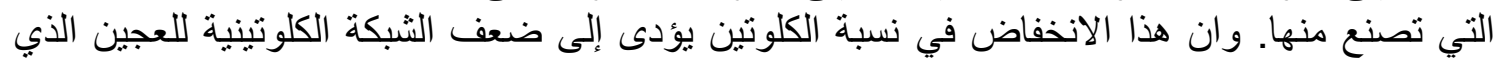

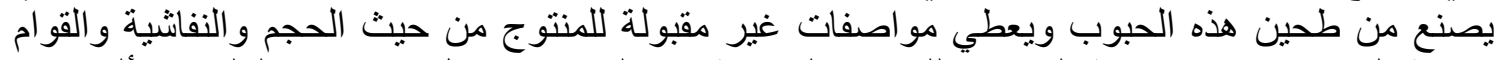

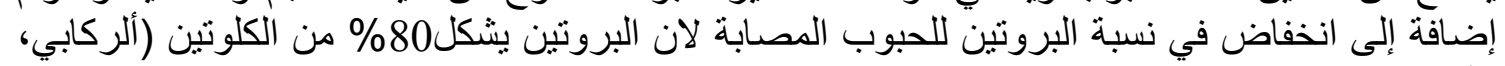

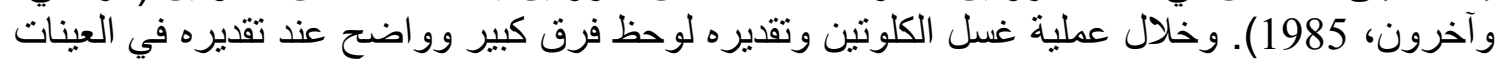

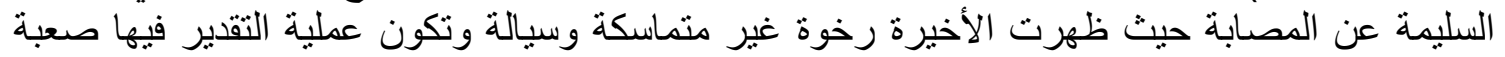

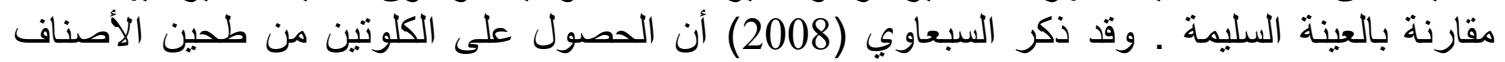

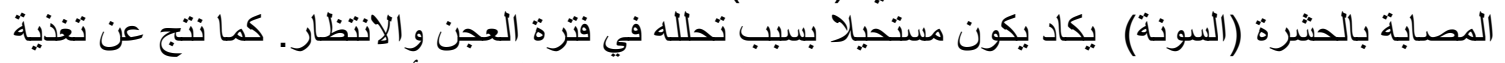

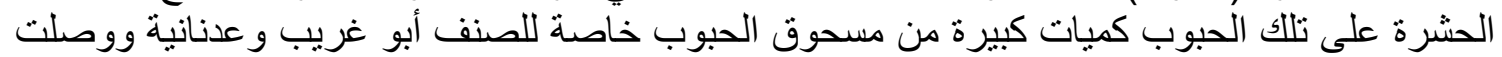

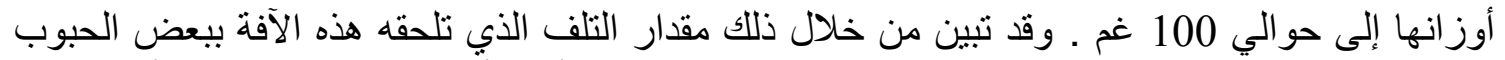

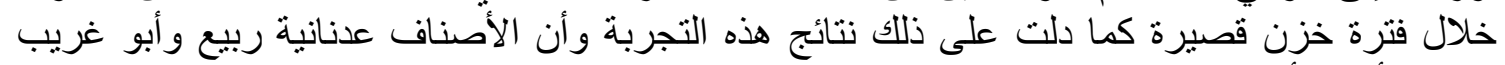

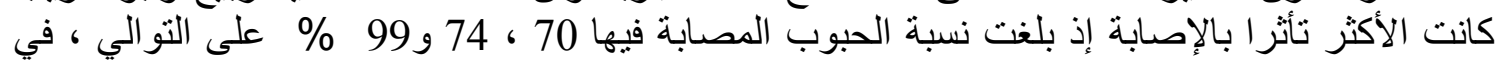

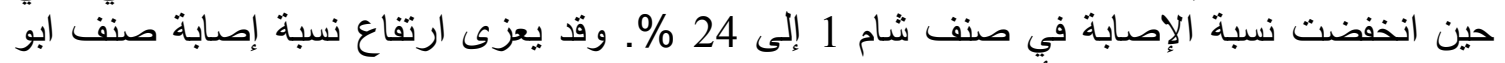

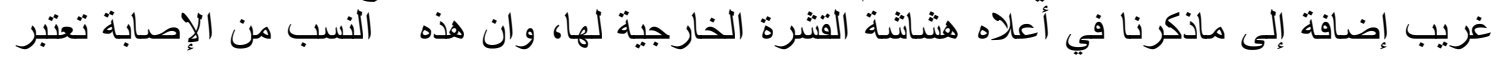

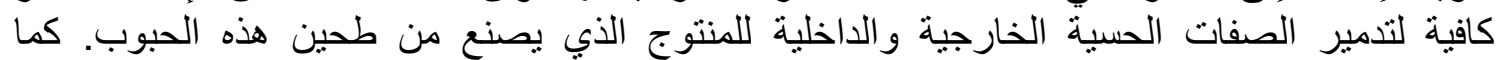

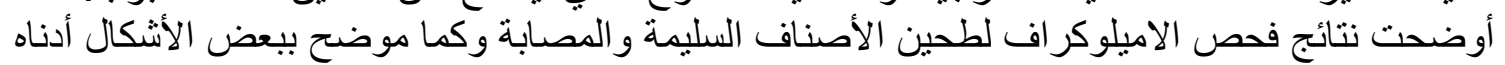

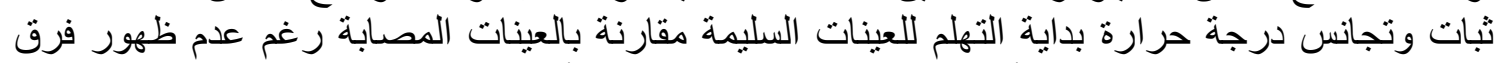

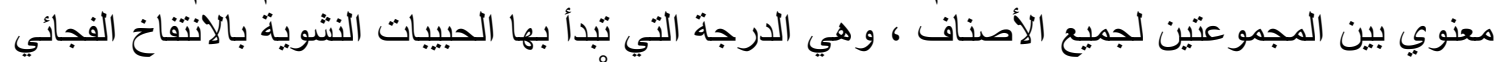

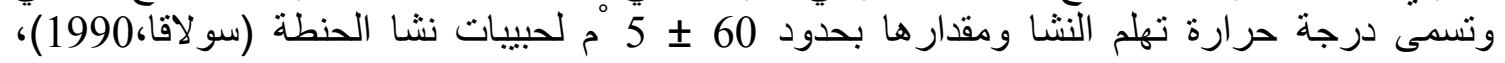

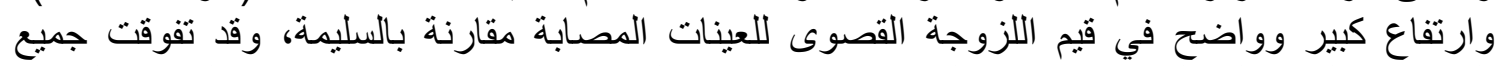

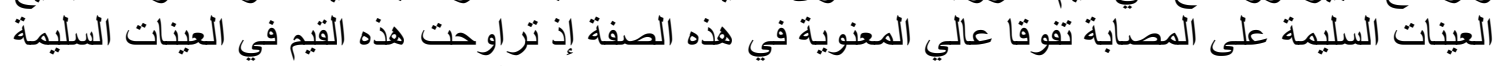

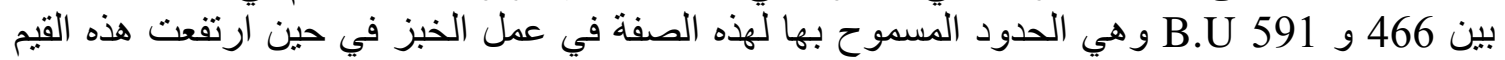

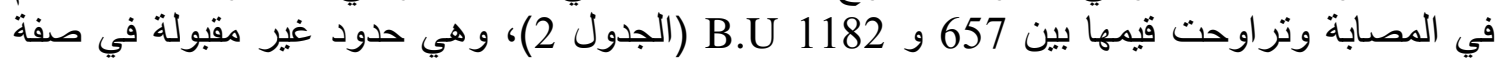

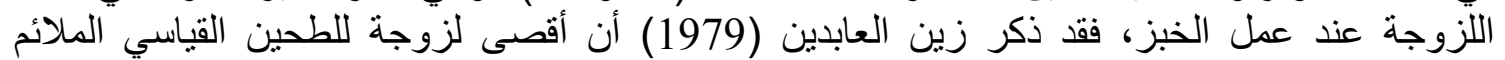

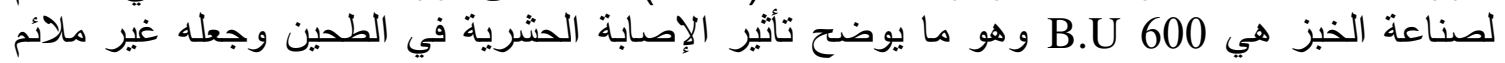

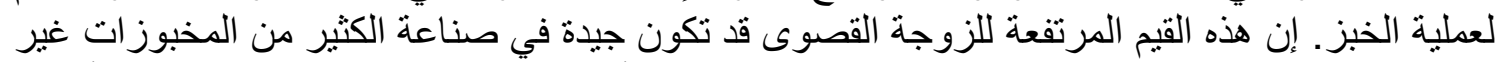

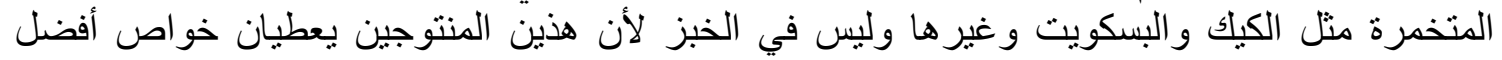

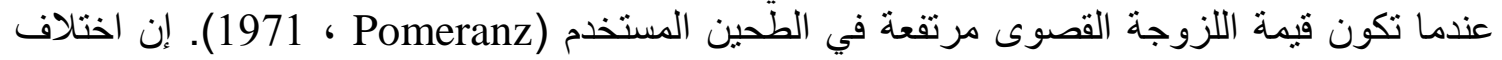

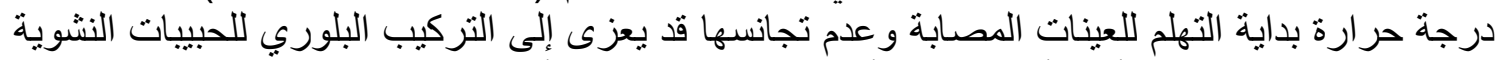

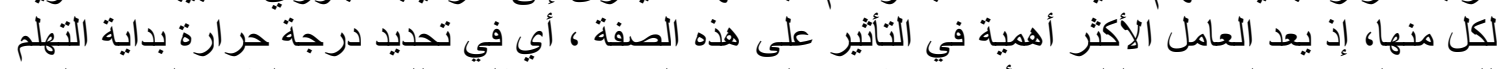

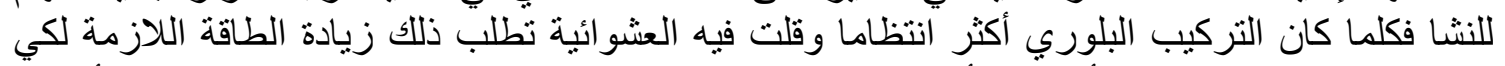

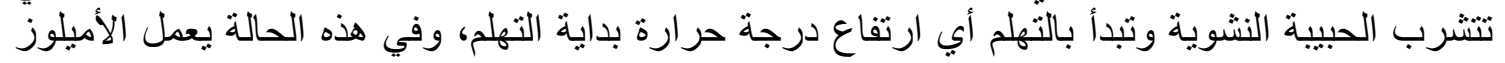

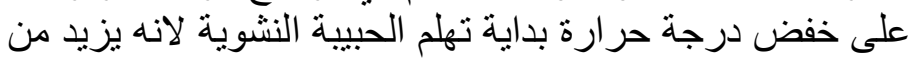


Mesopotamia J. of Agric.

Vol. (41) No. (1) 2013
ISSN: 2224-9796 (Online) ISSN: $1815-316 \mathrm{X}$ (Print)

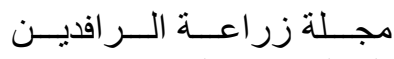
المجلد (41) العدد (1) 2013
المصابة Infested Samples
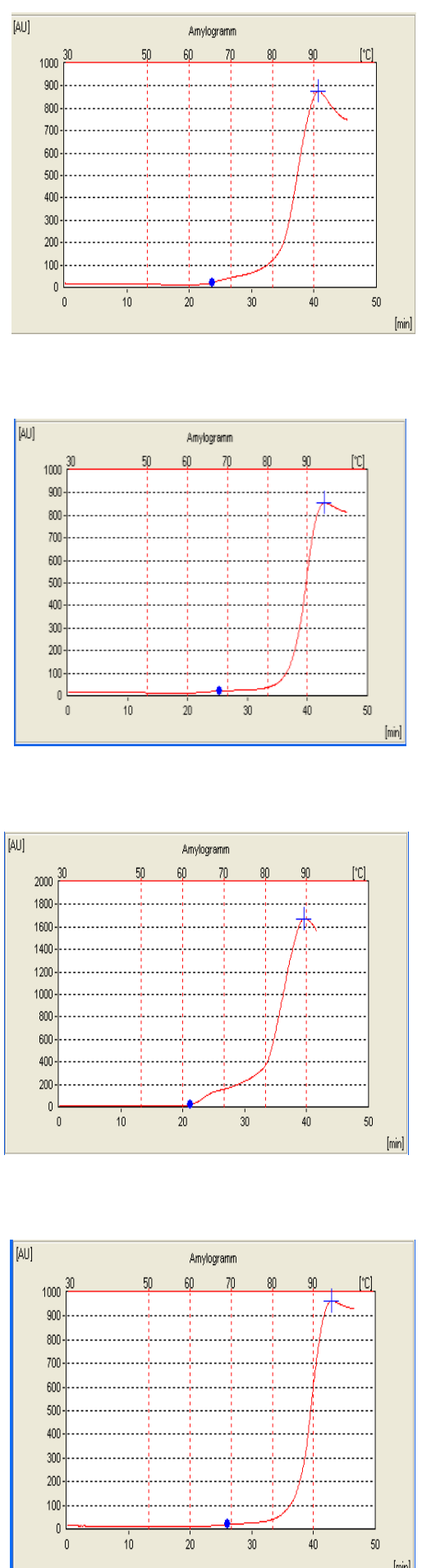

الشكل(1): نماذج لمنحنى الاميلوكر اف لبعض الأصناف من طحين الحبوب السليمة تقابلها المصابة.

Fig.(1).Amylograph curves of some samples of wheat flour for infested and sound varieties of grain.
Sound Samples السليمة

Sham6

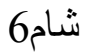

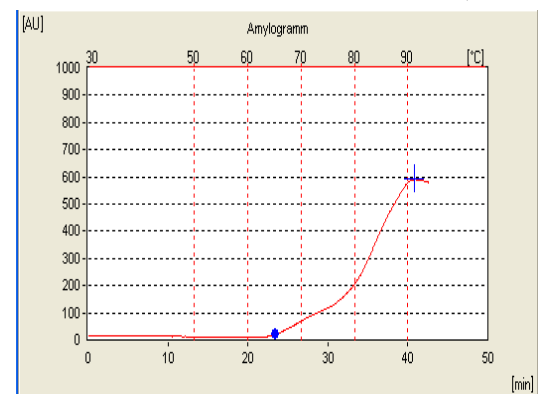

Abu-Graib أبو غريب
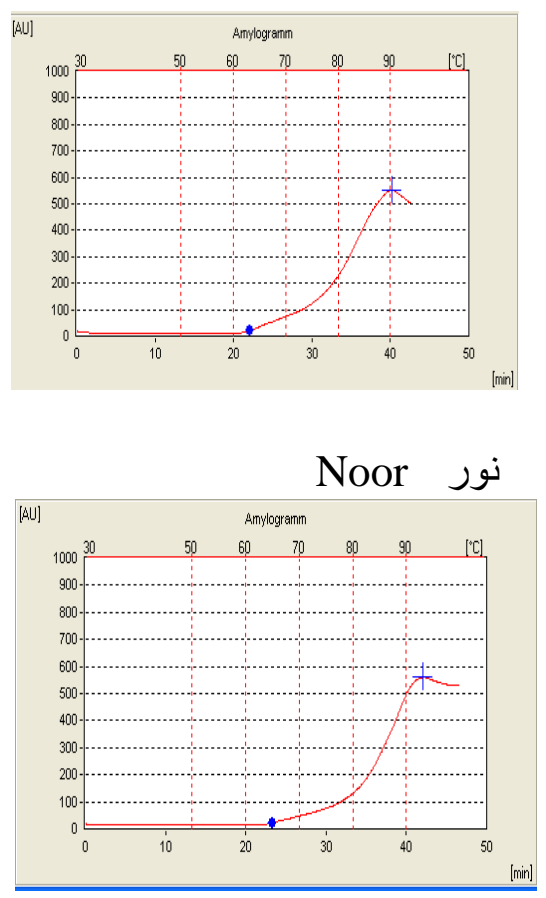

Adnania عدنانية

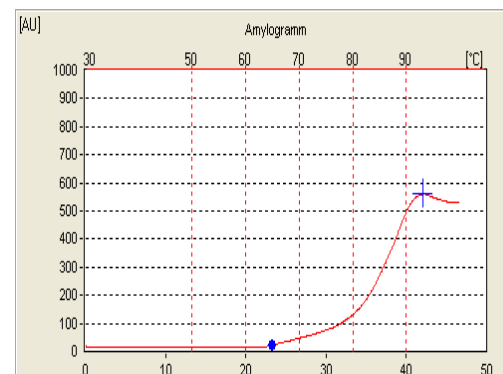
[nim| 
Mesopotamia J. of Agric.

Vol. (41) No. (1) 2013
ISSN: 2224-9796 (Online) ISSN: $1815-316 \mathrm{X}$ (Print)

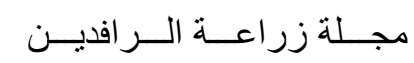
المجلا (41) العدد (1) 2013

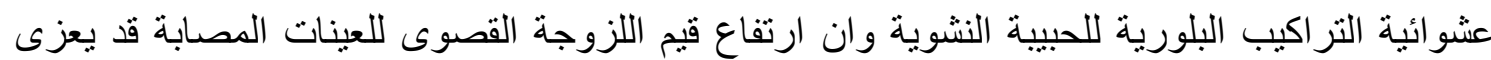

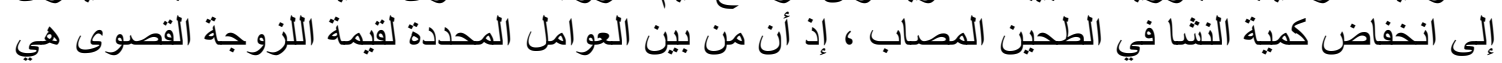

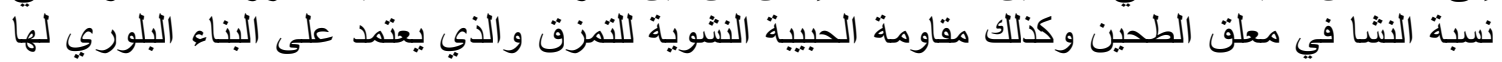
1994 Morrison)

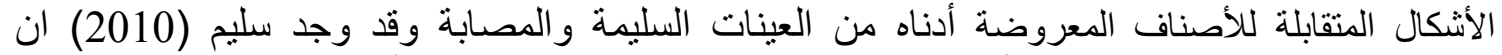

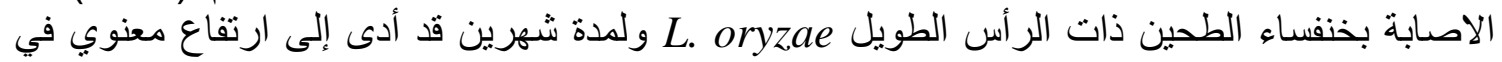

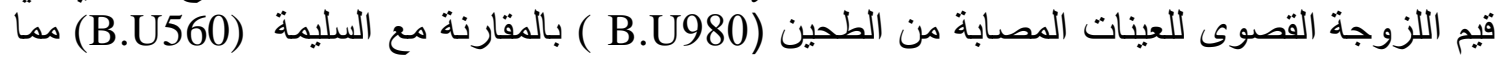

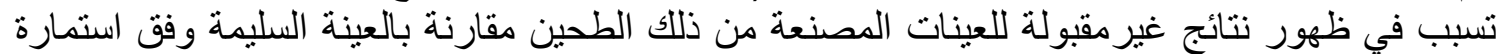

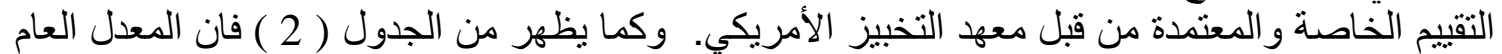
لقيم اللزوجة القصوى للعينات السليمة بلغ المصابة التي بلغت B.U مما يدل دلالة واضحة على مدى تأثير الإصابة الحشرية على مجمل

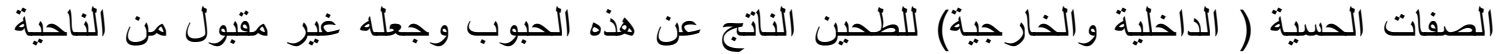

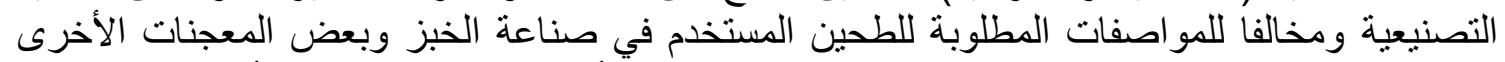

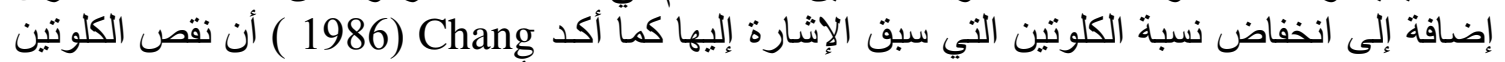
يعطي الطحين صفة السيولة ونقص المطاطية والتماسك خلال فترة 3 أنثهر من الإصنابة.

R. dominica لعشرة أصناف من الحنطة تحت ظروف العناف الحاضنة.

Table(2): Amylograph values of sound and infested samples by lesser grain borer to 10 varieties of wheat under incubator conditions.

\begin{tabular}{|c|c|c|c|c|c|c|}
\hline \multicolumn{2}{|c|}{$\begin{array}{l}\text { * B.U اللزوجة القصوى } \\
\text { Maximum viscosity }\end{array}$} & \multicolumn{2}{|c|}{$\begin{array}{l}\text { درجة حرارة التهلم القصوى } \\
\text { Gelatinization max. }\end{array}$} & \multicolumn{2}{|c|}{ 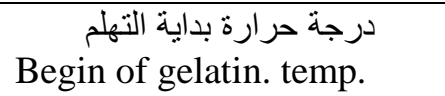 } & \multirow[t]{3}{*}{$\begin{array}{c}\text { الأصناف } \\
\text { Varieties }\end{array}$} \\
\hline صل مابة & السليمة & كسابة & السليمة & 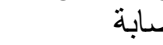 & السليمة & \\
\hline Infested & Sound & Infested & Sound & Infested & Sound & \\
\hline 757 & 466 & 93.0 & 91.4 & 66.0 & 64.4 & Sham 1 شام1 \\
\hline 963 & 570 & 94.9 & 92.1 & 75.8 & 63.0 & Sham 4 شام4 \\
\hline 878 & 591 & 91.0 & 91.3 & 65.6 & 65.1 & شام 6 Sham 6 \\
\hline 858 & 551 & 94.1 & 90.3 & 68.0 & 63.1 & أبوغريب \\
\hline 1013 & 560 & 92.1 & 93.0 & 64.5 & 62.0 & تموز2 2 July \\
\hline 657 & 540 & 92.4 & 89.4 & 59.8 & 61.8 & Noor \\
\hline 1182 & 545 & 92.9 & 89.8 & 56.4 & 65.5 & Iba'a $955^{95 \text { إباء }}$ \\
\hline 979 & 575 & 91.5 & 92.1 & 65.1 & 65.0 & Iba'a 99 \\
\hline 757 & 525 & 93 & 90.6 & 66.0 & 65.9 & Om-Rabee \\
\hline 966 & 560 & 94.4 & 93.0 & 69.0 & 65.0 & Adnania عدنانية \\
\hline b 901 & a 548.4 & -- & -- & 65.62 & 64.08 & المعدل Mean \\
\hline
\end{tabular}

*المتوسطات لصفة اللزوجة القصوى للعينات السليمة اختلفت بشكل عام وكلي معنويا عنها في المصابة عند مستوى احتمال 0.01 .

*The averages of Maximum viscosity of sound samples were differed significantly with the infested samples at the level of 0.01 .

وذكر Amjad و آخرون (2009) أن إصـابة الحنطـة بخنافس الطحين من الجنس Tribolium أدى إلى الـى

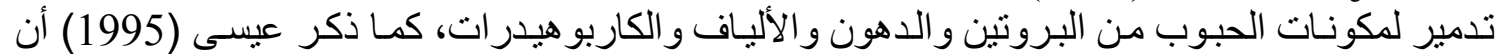

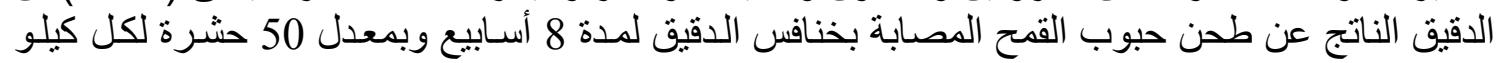

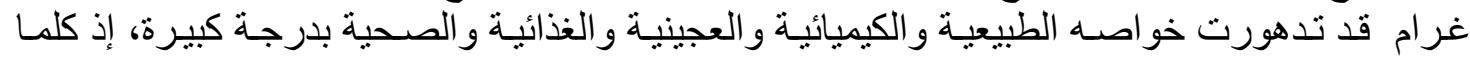




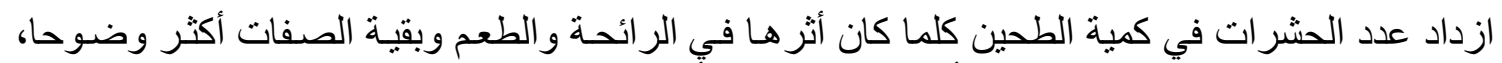

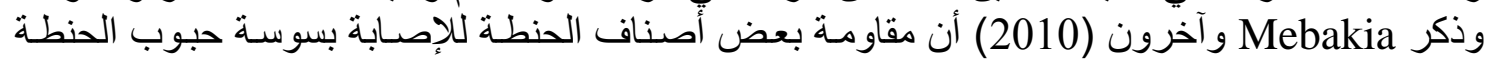
Sitophilus granarius

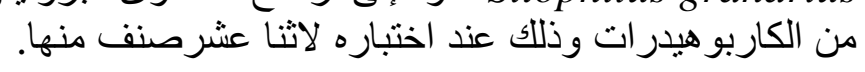

\title{
COMPARING THE INFESTATION EFFECT BY LESSER GRAIN BORER (RHIZOPERTHA DOMINICA) ON 10 WHEAT VARIETIES BEFORE AND AFTER THRESHING
}

\author{
O .S .Mohammad
}

College of Agriculture \& Forest. Univ. of Mosul, Iraq.

Osa1953@Yahoo.com

\begin{abstract}
This study was conducted to test the ability of the lesser grain borer to attack 10 varieties of wheat and caused any injury to the grain stored in their ears, and to compare that with the infestation of the released seeds of the same varieties. The results showed that the beetle failed to cause any damage to the first group of wheat, although the process of re-infection were repeated three times, and that the incidence of the insect for all varieties of wheat stored in the ears was zero \% , and that all insects were dead within a maximum of 10 days after putting them on the ears, while the percentage of germination of the stored grains reached $75.2 \%$ as an average.,while it was decreased for with an average of $32.3 \%$. It also resulted in injury insect to a significant decrease and clear the proportion of gluten in the flour of infected seeds with an average of $19.9 \%$ as compared with that stored in the ears showing an average of $35.2 \%$. On the other hand the percentage of infection for the released seeds reached $52.3 \%$ as an average., as confirmed by the amylograph test the existence of significant differences between the values of the maximum viscosity were very high in the infected samples giving an average of 901 B.U while it was in the sound 548.4B.U.,such differences for some of the sensory qualities of grain exposed to insect injury made it unacceptable from the standpoint of work manufacturing laboratory bread or some other pastry.

Keywords:Lesser grain borer, wheat varieties, Rheological properties.
\end{abstract}

Received : $25 / 9 / 2011$ Accepted 13/2/2012

$$
\begin{aligned}
& \text { ابن كثير ، إسماعيل بن عمر .(1986). تفسير القران العظيم ، دار المعرفة بيروتـ لبنـان، الطبعة الأول } \\
& \text { ج2 } 498 . \\
& \text { الر اوي، خاشع محمود و عبد العزيز خلف الله (1980). تصميم وتحليل التجارب الزراعية وزارة التعليم }
\end{aligned}
$$

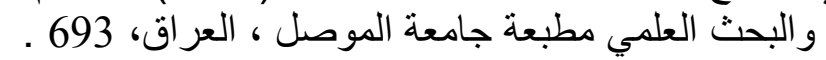

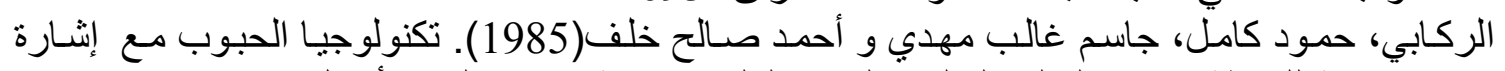

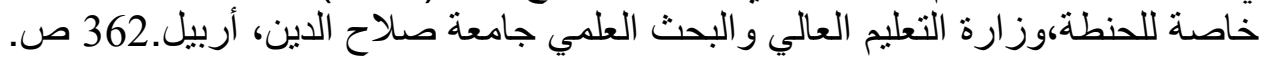


زين العابدين ، محمد وجيـه (1979). در اسـة تثبيت المو اصفات القياسية للطحين الملائم لإنتـاج الخبز

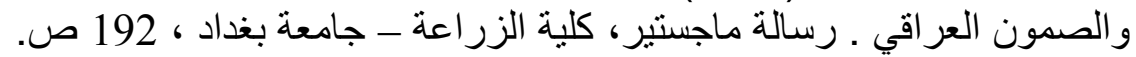

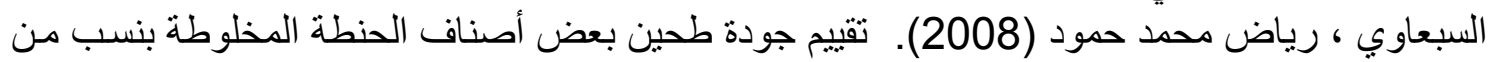

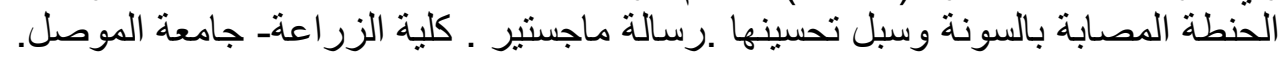

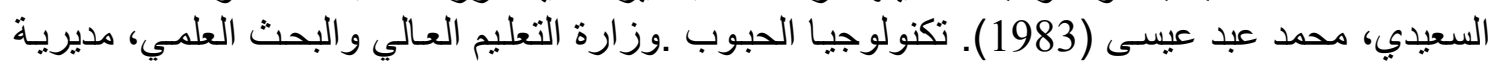

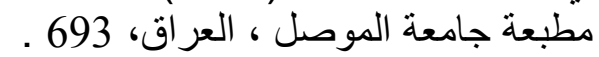

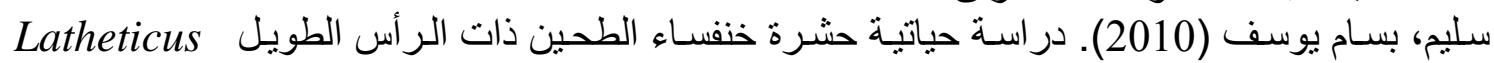

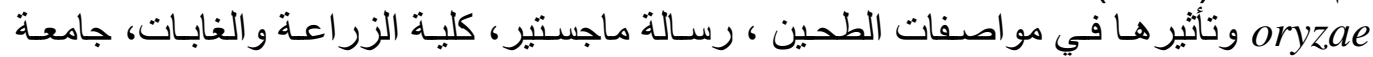

$$
\text { الموصل، } 80 \text { صن. }
$$

سو لاقا ، أمجد بويا (1990). الخبز و المعجنات ، مطابع التعليم العالي، جامعة الموصل، 360ص، ولك.

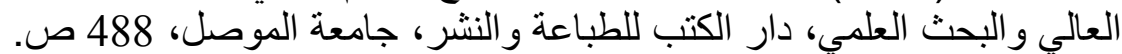

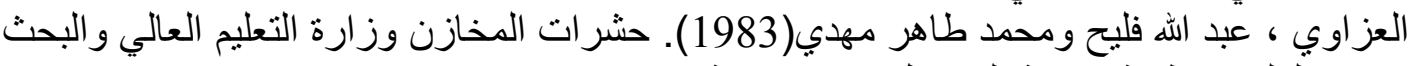
العلمي مطبعة جامعة الموصل ولن 484 صفحة.

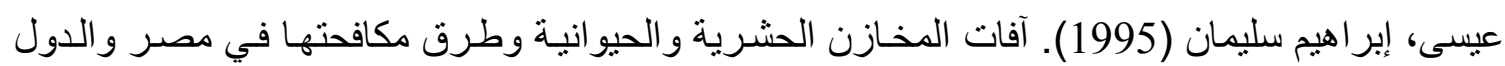

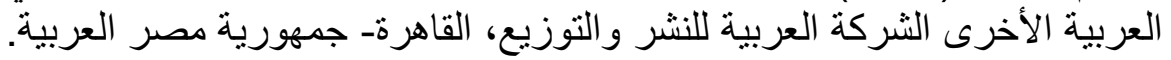

Anonymous (1976). Approved Methods of American Association of Cereal Chemists, A.A.C.C St. Paul., MN., USA.

Anonymous (1983). Approved Methods of American Association of Cereal Chemists.10 ${ }^{\text {th }}$ Ed. Methods 38-10, A.A.C.C. St. Paul., MN., USA.

Amjad, A., Muhammad, S., Shamadad, K. and G. H. Abro (2009). Reaction of certain wheat varieties to the action of red flour beetle, Tribolium castaneum (Herbst) (Coleoptera) under insectary conditions. Pakistan Journal of Zoology,. 41 (1), pp. 51-56.

Belitz, H. W; Grosch and P. Schieberle (2009). Food Chemistry. $4^{\text {th }}$ ed. Springer, USA.

Chang,O.K.(1986). Lipid- protein interactions in wheat flour dough, gluten and protein fractions. Cereal Foods World, 31; 242-254.

Duong, T.N. (2006). Analysis Of The Behaviour Of $R$. dominica (F.) Towards Host Volatile. Natural Resources Institute, University of Greenwich.

Fogliazza.D. and Pagani, M. (2003). Stored product pests affecting wheat and flour quality. Tecnica-Molitoria, 54: 897-903

Hoseney, R.C. (1986) Principles Of Cereal Science and Technology. American Association Of Cereal Chemists, St . Paul, Minn . USA.

Mebarkia, A., Rahbé Y., Guechi A., Bouras A. and M. Makhlouf (2010). Susceptibility of twelve soft wheat varieties to Sitophilus granarius (L.) (Coleoptera: Curculionidae). Agriculture Biology Journal North America, 1(4): $571-578$.

Morrison, W. R. (1994). Analysis of cereal starches in : Modern Methods Of Plant Analysis, New Series, VOI .14, Seed Analysis . F . Linskens and J.

F. Jackson, Eds. Spring -Verlay Heidelberg.

Pomeranz, Y. (1971). Wheat Chemistry and Technology. Published by the American Association of Cereal Chemists. In Corporate. St. Paul. Minnesota: 217-235.

Pomeranz, Y.(1988 ). Wheat chemistry and technology.Vol.102. American Association of Cereal Chemists, st. Paul, Minn. USA. 
Mesopotamia J. of Agric.

ISSN: 2224-9796 (Online)

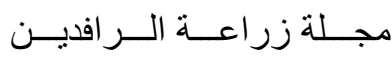

Vol. (41) No. (1) 2013

ISSN: $1815-316$ X (Print)

المجلد (41) العدد (1) 2013

Smith , L.W., Pratt Jr., I. Nii, A.P. Umina (1989). Baking and taste properties of bread made from hard wheat flour infested with species of Tribolium, Tenebrio, Trogoderma and Oryzaephilus. Journal of economic entomology 82 (4) 1254-1261

Stear, C. (1990). Handbook Of Bread Making Technology, Elsevier Applied Science., London. 